\title{
Nanoscale insight into the exfoliation mechanism of graphene with organic dyes: effect of charge, dipole and molecular structuret
}

\author{
Andrea Schlierf, $\neq^{\mathrm{a}}$ Huafeng Yang, $\neq^{\mathrm{b}}$ Elias Gebremedhn, $\neq^{\mathrm{c}}$ Emanuele Treossi, ${ }^{a}$ \\ Luca Ortolani, ${ }^{d}$ Liping Chen, ${ }^{c}$ Andrea Minoia, ${ }^{c}$ Vittorio Morandi, ${ }^{d}$ Paolo Samorì, ${ }^{\text {ee }}$ \\ Cinzia Casiraghi, ${ }^{\star b}$ David Beljonne*c and Vincenzo Palermo*a
}

\begin{abstract}
We study the mechanism of surface adsorption of organic dyes on graphene, and successive exfoliation in water of these dye-functionalized graphene sheets. A systematic, comparative study is performed on pyrenes functionalized with an increasing number of sulfonic groups. By combining experimental and modeling investigations, we find an unambiguous correlation between the graphene-dye interaction energy, the molecular structure and the amount of graphene flakes solubilized. The results obtained indicate that the molecular dipole is not important per se, but because it facilitates adsorption on graphene by a "sliding" mechanism of the molecule into the solvent layer, facilitating the lateral displacement of the water molecules collocated between the aromatic cores of the dye and graphene. While a large dipole and molecular asymmetry promote the adsorption of the molecule on graphene, the stability and $\mathrm{pH}$ response of the suspensions obtained depend on colloidal stabilization, with no significant influence of molecular charging and dipole.
\end{abstract}

Received 15th January 2013

Accepted 14th February 2013

DOI: 10.1039/c3nr00258f

www.rsc.org/nanoscale applications as dyes or as organic semiconductors for the fabrication of opto-electronic devices such as organic lightemitting diodes (OLEDs), ${ }^{\mathbf{9}, \mathbf{1 0}}$ organic photovoltaics, ${ }^{\mathbf{1 1 - 1 5}}$ flexible electronics ${ }^{16-18}$ and sensors. ${ }^{19}$ The optical and electronic properties of these NGs can be tuned by functionalizing their peripheries with electron accepting or electron withdrawing groups. ${ }^{\mathbf{2 0 - 2 8}}$ The side-groups can also improve their solubility not only in organic solvents but also in water, being the best solvent possible for large scale, environmentally friendly and cheap technological applications. In particular, several works have demonstrated the efficiency of water-soluble pyrenes to solubilize graphene sheets in water. The single or multiple functional moieties attached to the pyrene's periphery include negatively charged sulfonic groups, ${ }^{29-31}$ amines, ${ }^{31}$ carboxylic, ${ }^{32}$ butyric $^{33}$ or butylphenyl groups. ${ }^{34}$

While the efficiency of pyrene derivatives in exfoliating graphene is well known, the exact details of pyrene-graphene interactions, and more generally of NG-graphene interactions, are still unclear. By using a rather simplistic description of the exfoliation process, organic molecules such as these are often described as aromatic surfactants, "molecular wedges"32 with the apolar, aromatic part interacting with graphene through $\pi-$ $\pi$ stacking and the negatively charged part favouring sheet stabilization in solvents and hindering re-aggregation. ${ }^{35,36}$

The efficiency of amphiphilic pyrenes to interact with graphene has been attributed to their aromatic nature and to their electron donating/withdrawing ${ }^{29,37}$ character due to the difference in the dyes' electronegativity with respect to graphene. Yet, 
unexpectedly, a recent work by Green and co-workers found that an increase in the number of electron-withdrawing sulfonic groups yields a more modest graphene exfoliation in solution and that the adsorption efficiency of the stabilizer on graphene is not related to the protonation/deprotonation of pyrenes.

These results indicate that, while the amphiphilic nature of these organic molecules is fundamental to stabilize graphene in polar solvents, the simple "molecular wedge" model is not sufficient to describe both the interaction of aromatic molecules with graphene and their ability to exfoliate and solubilize graphite. The final efficiency of graphite exfoliation with small aromatic dyes ultimately depends not only on the thermodynamics of exfoliation, but also on the presence of local energy minima which will influence kinetics, and on the solventmolecule, graphene-molecule and graphene-solvent competitive interactions. ${ }^{38}$

To gain a thorough molecular scale understanding on the interaction process between graphene and the dye it is key to perform and compare nano-scale and multiscale experimental studies. In this work, we exfoliate graphene using a series of pyrene molecules functionalized in the peripheral positions with an increasing number of sulfonic groups. Our study contributes to derive the most important factors driving small molecule assisted graphene exfoliation and stabilization in suspension.

We compare two complementary processes that take place during graphene liquid phase exfoliation, namely:

(a) the adsorption of the molecules from a water solution to the surface of the bulk graphite.

(b) the exfoliation, induced by the molecules, of graphene sheets from the bulk graphite to a water solution.

Both processes are caused by the affinity between the molecule and graphene, and depend strongly on the molecular structure and processing conditions. The affinity of the dyes towards graphitic substrates and their efficiency in exfoliating and suspending graphene are studied using optical absorption and Raman spectroscopy, Atomic Force Microscopy (AFM) and Transmission Electron Microscopy (TEM), combined with $a b$ initio and molecular dynamics calculations.

We performed our comparative study by using different pyrene sulfonic acid sodium salts (Fig. 1). All these pyrenes are functionalized with side-groups featuring different steric hindrance, electronegativity and $\mathrm{pH}$ response to the respective
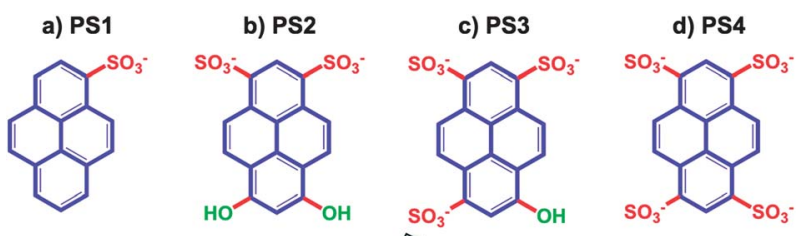

e)

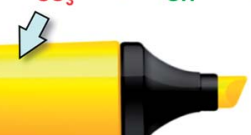

Fig. 1 (a-d) Chemical formula of the four pyrene derivatives under study. The moieties which shall be protonated/deprotonated are indicated in green. (e) Typical highlighter containing pyrene-based sulphonated dyes such as PS3. molecule. The molecules studied are: 1-pyrenesulfonic acid sodium salt (PS1), 6,8-dihydroxy-1,3-pyrenedisulfonic acid disodium salt (PS2), 8-hydroxypyrene-1,3,6-trisulfonic acid trisodium salt (PS3) and pyrene-1,3,6,8-tetrasulfonic acid tetrasodium salt (PS4). While PS1 and PS4 have already been demonstrated to be eligible surfactants for graphene liquid phase exfoliation, ${ }^{29-31,39}$ we introduce here also the more complex analogues PS2 and PS3 having electron accepting, sulfonic $\left(-\mathrm{SO}_{3}{ }^{-}\right)$groups and electron donating - $\mathrm{OH}$ groups, whose charge and dipole can be modified with $\mathrm{pH}$. We selected these $\mathrm{pH}$ sensitive derivatives because they are not only interesting for fundamental research, but also for practical applications due to their high quantum yield, excellent water solubility, ratiometric properties and lack of toxicity. ${ }^{40}$ PS3 for instance, also known as pyranine or solvent green 7 , is commonly used in commercial highlighters and soaps.

The extraordinary optical and chemical properties of these molecules such as their well-defined absorption and emission in the visible spectrum, tunable absorption, emission and molecular charge, and $\mathrm{pH}$ sensitive dipole, make them interesting materials to be combined with graphene. To provide precise, comparable and quantitative insight into the efficiency of these pyrene sulfonic sodium salts for the preparation of graphene, we carefully respected the same procedures for all derivatives including the solvent, sonication and excess dye removal parameters. Such a reliable comparison is not simply achieved by considering previously reported results as processing methods and characterization techniques largely vary and consequently do not allow for direct comparison. ${ }^{29-35}$

\section{Results}

\section{Properties of the free dyes}

Fig. 2a-d (red curves) show the different absorption spectra of the four molecules. Fig. 2 reveals that the absorption spectrum of pyrene is strongly influenced by side functionalization. The extreme tunability of pyrene optical absorption is well known, and was also exploited to create a "pyrene scale of solvents", to rank the polarity of solvents. ${ }^{41}$ PS1 and PS4 exhibit a well-defined optical absorption band structure, with three main sharp peaks similar to those of pristine pyrene; ${ }^{42}$ conversely, the presence of $-\mathrm{OH}$ groups in PS2 and PS3 greatly influences the electronic structure of the molecule, leading to broadened and red shifted peaks. The different absorption is not due to a change in molecular aggregation behaviour in solution, as proven by performing concentration dependent absorption studies (not shown); instead the $-\mathrm{SO}_{3}{ }^{-}$groups act as electron withdrawing units on the polyaromatic scaffold, while the $-\mathrm{OH}$ is a weak (mesomeric) electron donor. The push-pull action of the two groups results in a significant reduction of the lowest optical electronic excitation, thus moving the absorption peaks to longer wavelengths, ${ }^{42}$ an effect successfully used in the last few years to improve light absorption and efficiency in organic photovoltaic dyes. ${ }^{13}$

A further important consequence of the different functionalization is a change in the molecular dipole moment, which has been calculated in an implicit water environment using $a b$ initio methods. The calculation is performed at the b3lyp/6- 

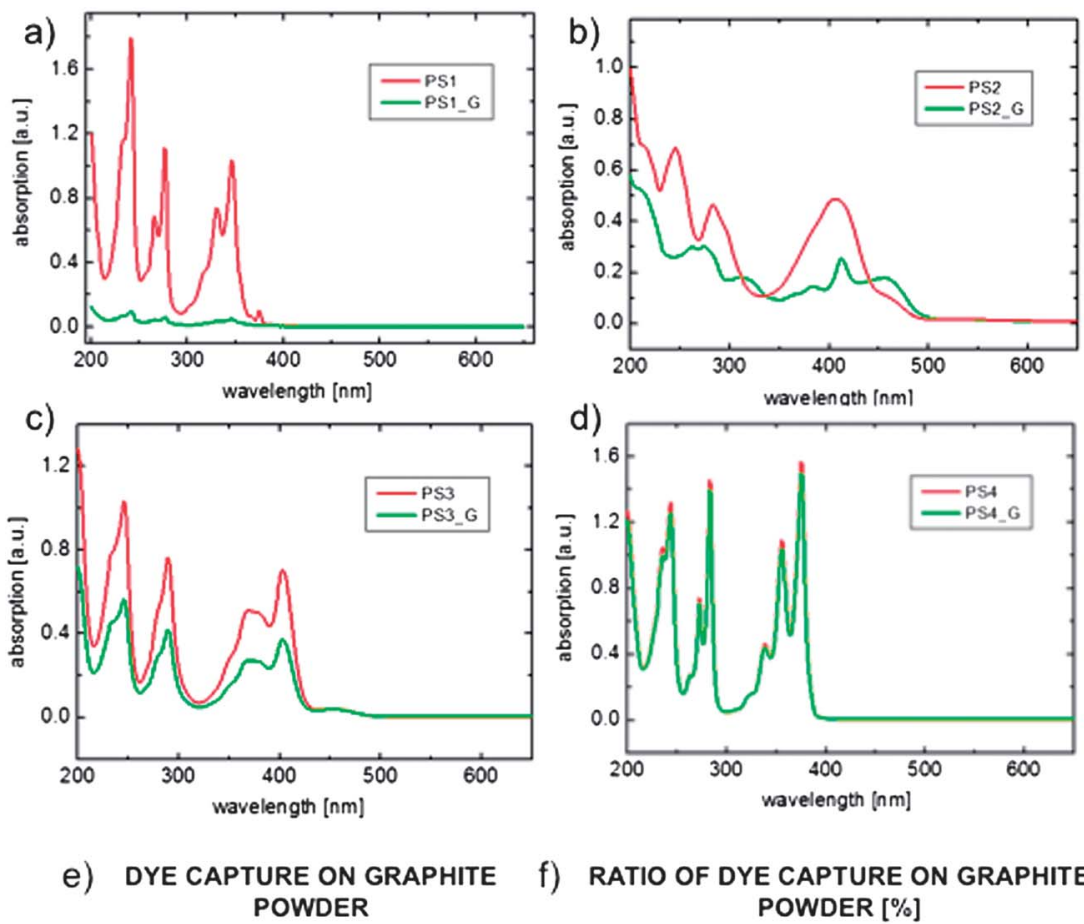

\section{f) RATIO OF DYE CAPTURE ON GRAPHITE POWDER [\%]}
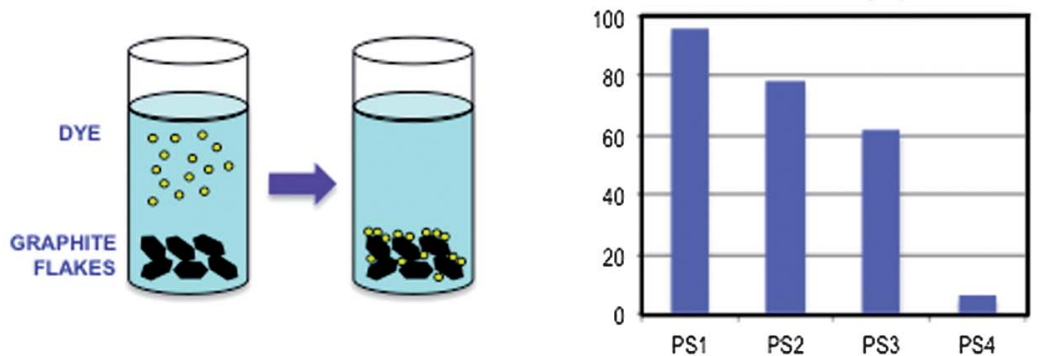

Fig. 2 (a-d) UV/VIS absorption spectra of aqueous solutions of PS1, PS2, PS3, PS4 dyes after stirring for 4 days in pure water (red curves) or in water in the presence of graphite powder (green curves). All samples contain the same initial dye concentration and ratio of molecules and graphite powder. (e) Schematic representation of "dye capture" from solution to the graphite substrate. (f) Ratio of molecules removed from solution upon exposure to graphite powder.

$31 \mathrm{~g}(\mathrm{~d}, \mathrm{p}) / \mathrm{PCM}$ level within the Gaussian 03 package. Regardless of the highly polar functionalization, the dipole moment is zero for PS4 as a consequence of the molecule's highly symmetric geometry. PS1 and PS3, which belong to the same symmetry group, show comparable molecular dipole moments (25.9 Debye for PS1 and 28.0 Debye for PS3). The dipole is instead strongly pronounced in PS2 (46.6 Debye), due to the presence of both electron-withdrawing groups in positions 1 and 3 and the electron pushing - $\mathrm{OH}$ groups in positions 6 and 8 .

The $-\mathrm{OH}$ group also adds another degree of freedom to control molecular properties: while the sulfonic groups are salts of strong acids and can be considered as negatively charged in any relevant $\mathrm{pH}$ conditions ( $\mathrm{p} K_{\mathrm{a}}$ for sulfonic acids is $<0$ ), the -OH groups in PS2 and PS3 are weakly acidic and their protonation state is highly sensitive to $\mathrm{pH}$ (for PS3 the $\mathrm{p} K_{\mathrm{a}}$ is 7.3, for the two $-\mathrm{OH}$ of PS2 the $\mathrm{p} K_{\mathrm{a}}$ are 7.33 and 8.53). ${ }^{40}$ The dipole of PS2 and PS3 dyes changes significantly with $\mathrm{pH}$ from acidic to basic conditions, decreasing from 46.6 to 25.0 Debye for PS2 and from 28.0 to 17.4 Debye for PS3. In both PS2 and PS3, basic conditions give rise to the characteristic, lower energy absorption band that is attributed to the deprotonated species (Fig. S1 in $\left.\mathrm{ESI}^{\dagger}\right)$. The absorption of the protonated species, which is blue shifted as compared to the deprotonated one, can be fully recovered by tuning the $\mathrm{pH}$ to acidic conditions. Thus, the acid/ base forms of those molecules can easily be discriminated by considering their different absorption spectra, and have already been used to monitor pyrene interactions with reduced graphene oxide (RGO) sheets in solution. ${ }^{43}$

\section{Dye adsorption on graphite powder}

The first stage of any exfoliation process is the interaction of the exfoliating agents with the pristine, bulk graphite powder. Before comparing the ability of the selected molecules to exfoliate graphene sheets from graphite, we estimated their potential to interact with graphitic substrates by monitoring their spontaneous physisorption from solution onto bulk graphite, which typically takes place in few hours. As previously reported, the spontaneous adsorption process of PS1 on carbon nanotubes reaches equilibrium after $12 \mathrm{~h}$ exposure time. ${ }^{\mathbf{4 4}}$

In our experiment, a fixed amount of graphite powder (Aldrich) was immersed in an aqueous solution of the studied 
molecules. Gentle stirring was applied to avoid artifacts due to diffusion limits; samples were stirred for four days to ensure efficient adsorption. In the absence of sonication the exfoliation does not take place, although the molecules having a higher affinity for graphite may initiate their adsorption process on the surface of the graphite powder. Therefore, the graphite powder acts as a trap for the molecules in solution, thereby capturing the dyes from the solution. The amount of this "dye capture" process was calculated by simply observing the decrease in optical absorption intensity upon exposure time.

Fig. 2 compares the absorption spectrum in water for the four molecules, after stirring in the presence (green lines) or absence (red lines) of graphite. As expected, stirring without graphite does not change the original absorption spectrum of the dyes; conversely, in the presence of graphite spontaneous adsorption from solution to the graphitic surface is observed, with an efficiency inversely proportional to the number of $-\mathrm{SO}_{3}{ }^{-}$ groups present (Fig. 2f). The fraction of molecules adsorbed on graphite has been calculated by monitoring the change in the main absorption peak for each molecule (at $346 \mathrm{~nm}, 408 \mathrm{~nm}$, $404 \mathrm{~nm}$ and $375 \mathrm{~nm}$ for PS1, PS2, PS3, PS4 respectively). For the mono-substituted derivative PS1, most of the molecules are adsorbed on the graphite surface after 4 days. PS4, having four sulfonic groups, did not show a change in free dye concentration after four days, indicating significantly lower affinity for graphite powder than PS1. Both molecules exposing hydroxyl groups show similar behaviour in spontaneous adsorption, with adsorption efficiencies between those of PS1 and PS4.

The change is not linear with the number of $-\mathrm{SO}_{3}$ groups on the pyrene core (decrease in absorbance of dye captured is $18 \%$ going from one sulfonic group to two, $16 \%$ from two to three, and $56 \%$ going from three to four). A quantitative correlation between the amount of dye adsorbed and the graphite surface available cannot be derived directly with the drop in dye concentration: the dyes tend to form a multilayer on graphitic substrates rather than adsorbing into homogeneous monolayers, as it will be evident from the results of the following sections. However, this qualitative insight reveals that the more sulfonic groups present on the pyrene core, the less favorable the interaction with graphite powder.

\section{Exfoliation and stabilization of graphene in suspension}

Previous works demonstrated that both PS1 and PS4 serve as effective agents in exfoliating graphene by sonication..$^{29-31,39}$ To compare the exfoliation efficiencies of the four dyes under study, a concentrated aqueous solution of each molecule was mixed with graphite powder (Aldrich) and sonicated in a water bath. A rough estimation of the amount of suspended graphitic material is obtained by comparing the total light absorption at $650 \mathrm{~nm}$. Given that all dyes used are transparent at $\lambda>500 \mathrm{~nm}$ (Fig. 2), the increase in absorption can be attributed uniquely to light absorption and scattering of the exfoliated material for any wavelength $>500 \mathrm{~nm}$. Optical absorption spectroscopy showed that the exfoliation yield, i.e., the quantity of suspended material, increases with sonication time (Fig. S2 in ESI $\dagger$ ).

Even if relevant exfoliation shall be observed after few hours, extensive sonication was performed to monitor the increase of solubilized material with time. After sonication all four solutions contained well-suspended and dark graphitic material (showing a light shade of the typical dye colour, Fig. 3a). Exfoliation in $\mathrm{H}_{2} \mathrm{SO}_{4}$ solutions containing as well $-\mathrm{SO}_{3}{ }^{-}$charged a)

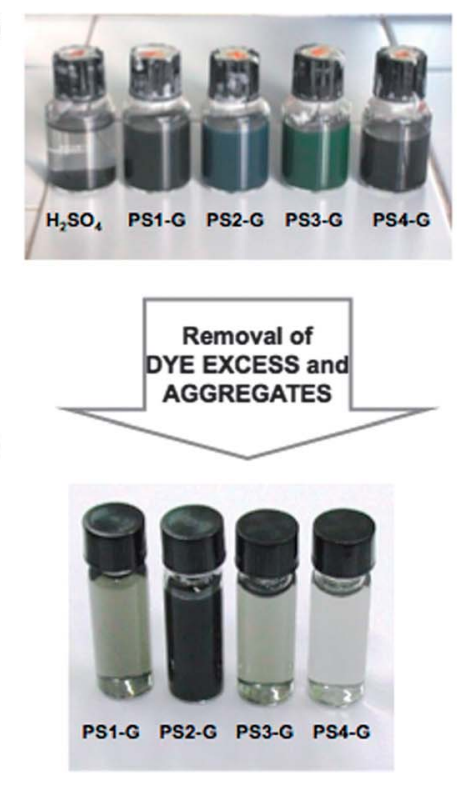

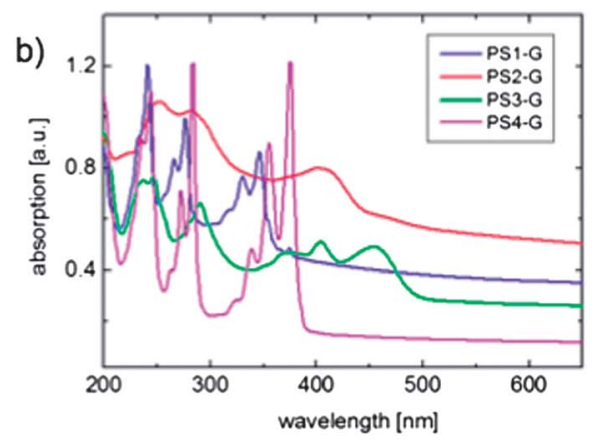

d)

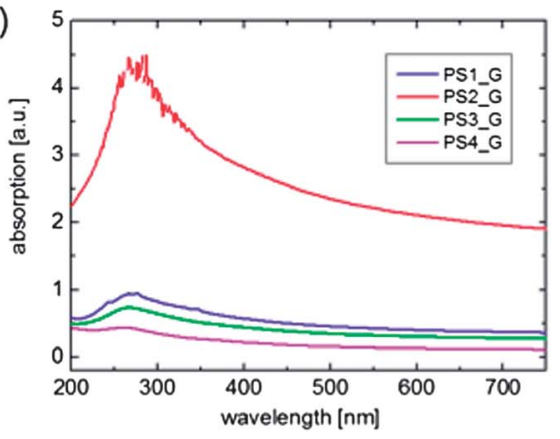

Fig. 3 (a) Picture of the four dye solutions after sonication with graphite and centrifugation. (b) UV/VIS absorption spectra of the suspensions (samples diluted 1 : 20 for measurement). Pyrene concentration $c=10^{-4} \mathrm{~mol} \mathrm{~L}^{-1}$. (c) Image of the suspensions after dye removal by washing and centrifugation. (d) Optical absorption of the solutions in (c) (no dilution). 
groups but no aromatic moiety (performed as a control experiment) did not yield any suspended material, as evident by the transparent colour of the suspension in Fig. 3a.

Fig. 3b and S2 $\uparrow$ clearly reveal that the increase in concentration strongly depends on the type of employed pyrene; for any sonication time tested, without further processing, the final concentration is the highest for PS2, followed by PS1, PS3 and PS4. Indeed, PS2 features a particular efficient action in dissolving graphite, while for the other derivatives PS1, PS3 and PS4 the graphite dissolution is inversely proportional to the number of sulfonic groups, as observed also in the "dye capture" experiment.

The freshly exfoliated suspensions contain a large excess of free dyes, as evident from the absorption spectra (Fig. 3b). The UV spectra show a superposition of the typical, well-defined pyrene band structure with the more uniform, slowly decaying light dispersion of graphene sheets in solution. The graphene was purified using a procedure based on multiple centrifugation steps, to remove excess dye and large graphite fragments, which was previously described in ref. 32 and 39 . After the washing and centrifugation procedure, the amount of material (both dye and graphene) present in suspension was significantly lower for all pyrene derivatives, even if PS2 suspensions were again the ones with the highest amount of solubilized graphene (Fig. 3c). Noteworthy, the typical absorption bands of pyrenes cannot be observed after the washing procedure, suggesting residual or adsorbed dye concentration being below the absorption spectrometer detection limit. All the suspensions obtained in this way were stable for several months, allowing efficient processing and further characterization.

\section{Morphology and composition of graphene-pyrene flakes}

The purified suspensions obtained by graphite sonication with the four different dyes were drop-cast on silicon substrates covered with $90 \mathrm{~nm} \mathrm{SiO}_{2}$ and characterized by TEM, AFM and Raman spectroscopy.

The distribution in sheet thicknesses and the presence of monolayers were measured with Raman spectroscopy by statistical analysis of the Raman 2D peak performed on several flakes. A significant part of the solubilized material was composed of single graphene sheets (up to $22 \%$ for PS3), with most of the remaining material composed of a few-layer graphene (50\% to $60 \%$ of flakes having less than 7 layers). A table with the statistical distribution of different layer thicknesses obtained for each molecule is reported in Fig. S3. $†$ The presence of a significant D peak at $1350 \mathrm{~cm}^{-1}$ and of a small peak at 1505 $\mathrm{cm}^{-1}$ was also observed by Raman, and attributed to the presence of the organic dyes (a more detailed study of the effect of PS1 on graphene Raman spectrum is reported in ref. 39).

The presence of graphene sheets together with adsorbed molecules was also confirmed by TEM analysis. Fig. 4 displays a TEM image of the typical sheets obtained using PS2, having lateral size of some hundreds of nanometers. Electron diffraction showed the typical hexagonal pattern of graphene, with reflections from $0.213 \mathrm{~nm}(0.246 \times \sqrt{ } 3 / 2)$ and $0.123 \mathrm{~nm}$ spaced lattice planes. In some flakes, the presence of a coating on
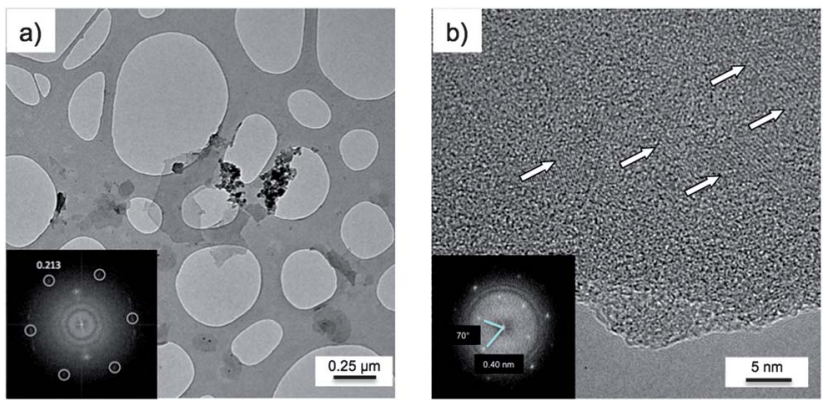

Fig. 4 (a) TEM image of graphene sheets exfoliated with PS2. In the inset, the typical SAED pattern of graphene is shown. (b) Zoom-in on a sheet, showing ordered areas with a packing different from the one observed for graphene (SAED measurements are shown in the inset).

graphene was observed by TEM. In such regions some superstructure appears in high-resolution images. Some patches of organized material are visible in Fig. $4 \mathrm{~b}$ as highlighted by the arrows.

Selected Area Electron Diffraction (SAED) measurements performed on the sheets showed that the hexagonal motif within the sheets was intact and undamaged by the interaction with the dyes, and that the dye molecules were organized in a crystal-like structure, with hexagonal symmetry and spacing corresponding to $0.44-0.45 \mathrm{~nm}$ (inset in Fig. 4b). The coating, though, showed different symmetry in different regions, having sometimes $0.40 \mathrm{~nm}$ reflections and cubic symmetry. TEM analysis did not provide statistical evidence, but this variation in symmetry and spacing could be explained by the presence of residual traces of crystallized solvent molecules. ${ }^{45}$

Fig. 5 shows typical AFM images of the graphene-pyrene composites spin coated on silicon. They display well-defined sheets, with polygonal shapes and sharp edges, having lateral sizes of some hundreds of nm. Nevertheless, AFM did not provide evidence for the presence of well-defined thicknesses; in fact, sheets with varying thickness (typically 2-4 nm) were obtained with all four dyes, in agreement with previous studies on PS1. ${ }^{39}$

The thickness observed is much higher than the theoretical one for monolayer graphene $(0.33 \mathrm{~nm})$; given that, as seen by Raman, the majority of flakes are $<7$ layers, the high thickness measured by AFM confirms the presence of a layer of adsorbed dye molecules on the graphene sheets, in agreement with Raman and TEM data.

\section{Influence of molecular polarity on graphene-pyrene interactions}

The main goal of our work is to provide a better understanding on the driving force for graphite exfoliation and stabilization with $\mathrm{pH}$ sensitive, water-soluble pyrene derivatives. Although the dye adsorption on graphite is simply correlated to the number of sulfonic groups on the aromatic core (Fig. 2), the highest concentration of suspended material has been obtained with the dihydroxyl-disulfonic derivative PS2, not the 
a)

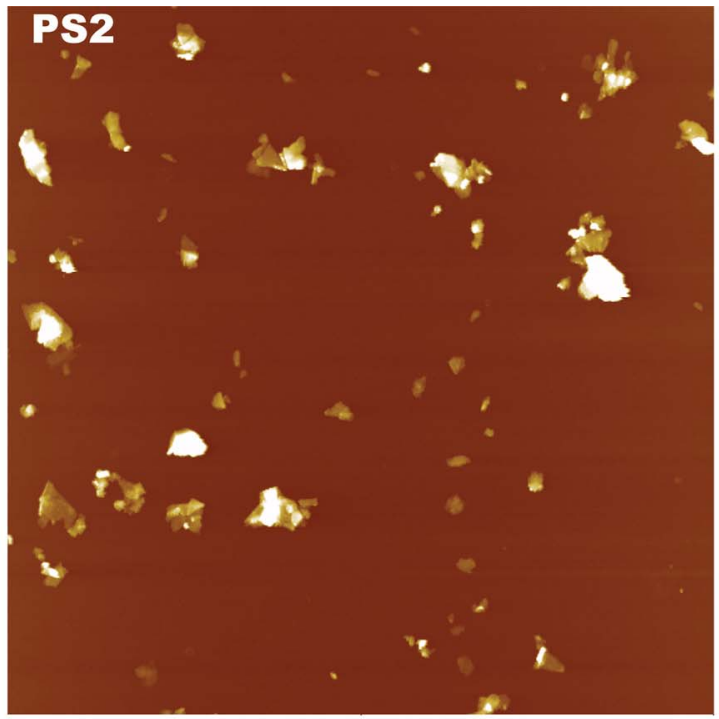

b)

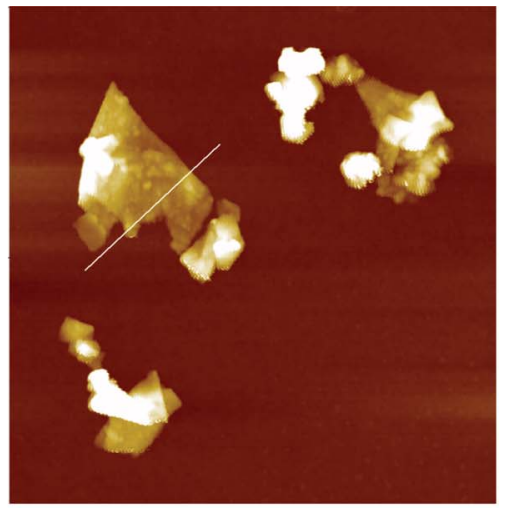

c)

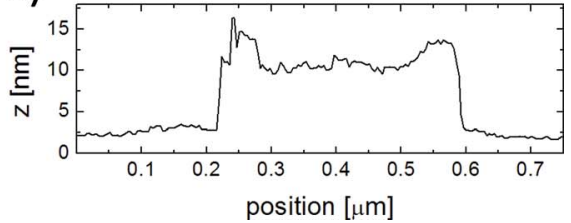

Fig. 5 (a) Topographical AFM image of PS2 suspension deposited by drop-casting on $\mathrm{SiO}_{2}$. Image width $10.0 \mu \mathrm{m}$. Z-range, $50 \mathrm{~nm}$. (b) Zoom-in showing the flake surface. Image width $3.3 \mu \mathrm{m}$. (c) Height profile taken along the white line in (b). $Z$-range, $30 \mathrm{~nm}$.

monosulfonic derivative PS1, excluding thus a simple effect on exfoliation of the steric demand of the side-groups.

As described above, PS2 has the highest dipole moment resulting from its molecular geometry with both bulky electron-
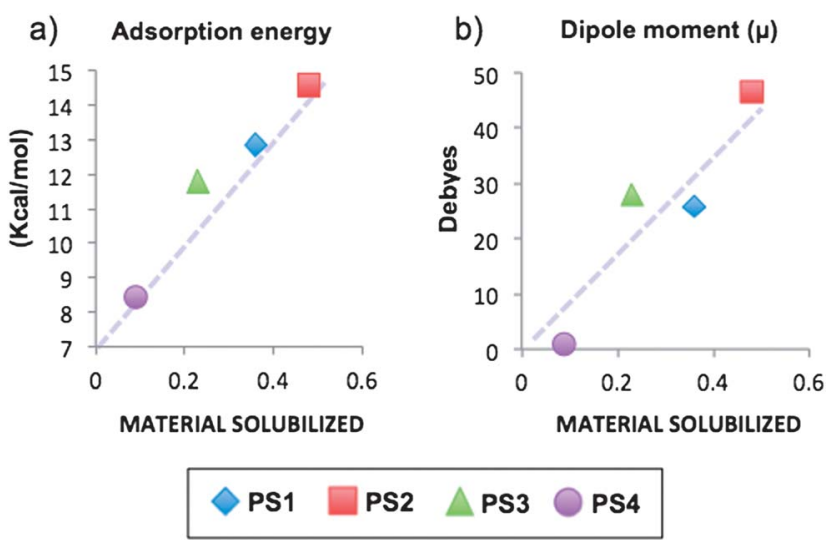

C) Number of polar groups
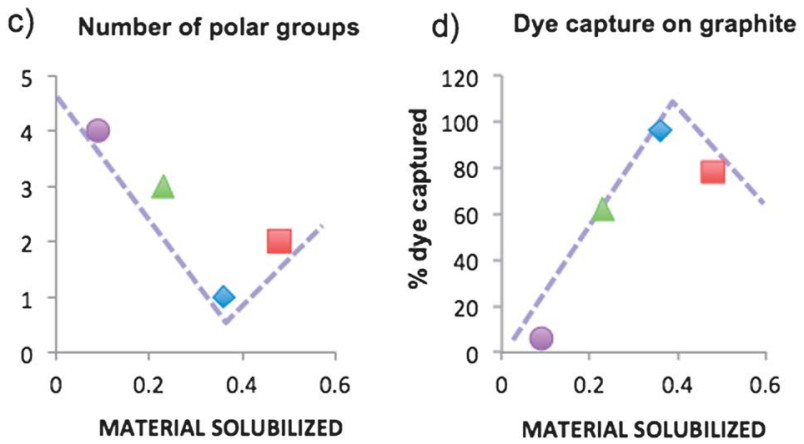

Fig. 6 Correlation between exfoliation efficiency and (a) calculated adsorption energy, (b) calculated molecular dipole, (c) number of charged groups on each molecule, (d) tendency of each molecule to adsorb on bulk graphite. The $X$ axis reports the amount of solubilized material, measured experimentally from UVIVIS absorption. The dashed lines are a reference for the eye. acceptor $-\mathrm{SO}_{3}{ }^{-}$groups in positions 1 and 3, and two smaller electron-donor-OH groups in positions 6 and 8 (Fig. 1). In Fig. 6 we correlate for each molecule experimental data and calculated molecular properties.

For all graphs, the $X$-axis reports the amount of material transferred from graphite into the suspension with sonication (quantified by the optical absorption at $650 \mathrm{~nm}$ ). The concentration of graphitic material in suspension is then plotted against the adsorption energy calculated by modelling (Fig. 6a, from force field calculations, see below) the molecular dipole (Fig. 6b, from DFT calculations), the number of polar, charged groups (Fig. 6c) and the tendency of each molecule to adsorb on bulk graphite (Fig. 6d, obtained from Fig. 2f).

The amount of exfoliated material ( $X$-axis) shows no clear correlation with both the number of polar groups present on the molecule (Fig. 6c), and the tendency of each molecule to adsorb on bulk graphite (Fig. 6d). Instead, a linear correlation with the molecule-graphene adsorption free energy is observed, Fig. 6a. The correlation with the molecular dipole is less clear; in particular, even if PS1 and PS3 dyes share the same group symmetry $\left(C_{\mathrm{s}}\right)$ and have a comparable dipole (25.9 D vs. $\left.28.0 \mathrm{D}\right)$, they do not yield the same concentration of exfoliated material. Experimental evidence in Fig. 3 shows that PS3 (which has a stronger acceptor-donor character due to the presence of one $-\mathrm{OH}$ and 3 sulfuric groups, but also features a large steric hindrance) performs worse than PS1. The lower steric hindrance of PS1, more than the strongest polarization of the PS3 aromatic core, is a factor important (but not sufficient) for successful exfoliation.

The correlations of physical properties with the amount of exfoliated material show that the pyrene derivative with both the highest molecular dipole and largest adsorption energy yields the most exfoliated material in suspension. However, a large molecular dipole moment is not enough for efficient graphene exfoliation, otherwise graphene would simply 


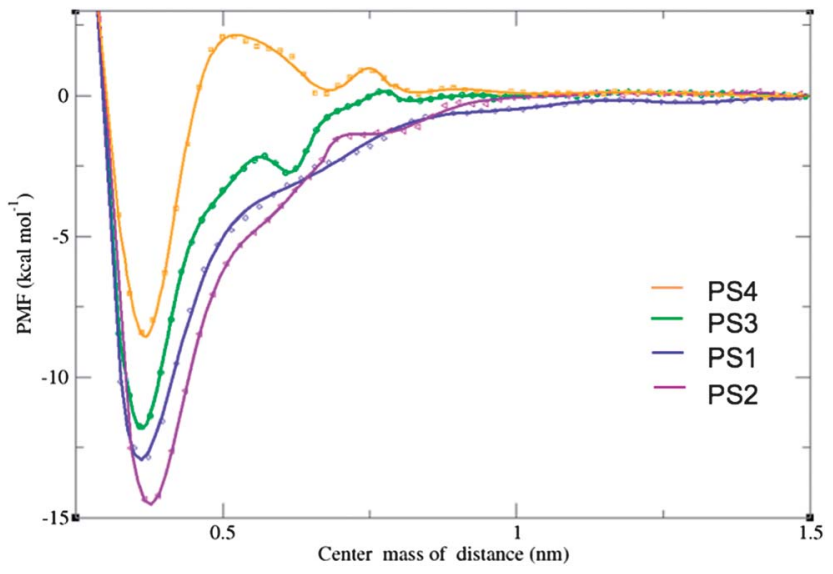

Fig. 7 Potential of mean force (PMF) vs. graphene-dye distance, calculated from umbrella pulling simulation technique.

exfoliate with highly polar solvents; the presence of the pyrene core, strongly interacting with graphene through $\pi-\pi$ interactions, plays of course a major role.

To better unravel the graphene-pyrene interaction, the adsorption process of each molecule on the surface of graphene in an explicit water medium was simulated using Molecular Dynamics simulations (see Simulation details section). The respective molecule was placed in a box, at a starting perpendicular distance of $1.8 \mathrm{~nm}$ from the basis plane consisting of a graphene sheet, and the box was then filled with water molecules. Periodic boundary conditions were maintained in all cases. An umbrella pulling simulation technique (see Simulation details section) was used to calculate the potential of mean force (PMF) of the adsorption process within several sampling windows. The free energy of adsorption of each molecule was determined from the resulting PMF curves.

Fig. 7 shows the PMF curves obtained for all four molecules. In contrast to PS1 and PS 2 that show a single global minimum in free energy, two local minima are identified on the PMF as the PS3 and PS4 molecules approach the graphene surface that could potentially act as kinetic traps. In particular, the calculations indicate that an energy barrier of a few $\mathrm{kcal} \mathrm{mol}^{-1}$ has to be overpassed to allow for close contact between PS4 and graphene. In order to gain insight into the 'landing on graphene' mechanism, snapshots at different stages of the molecular adsorption of PS2 and PS4 are reported in Fig. 8a and Fig. 8b, respectively.

As the PS2 dye adsorbs onto the surface of graphene, distinct structural features can be observed. While at large distance (position A), the molecule-graphene orientation is not relevant, at closer distance the molecule tilts, approaching the surface with the $-\mathrm{SO}_{3}{ }^{-}$side group pointing away from the graphene surface, as expected for an amphiphilic surfactant (B). However, once the molecule is at close distance from graphene $(\mathrm{C})$, the molecule slides and orients parallel to the surface, regardless of the presence of the charged sulfonic groups (D), maximizing $\pi-$ $\pi$ interactions as already observed for similar molecules in vacuum. ${ }^{8}$ In the case of PS4, the adsorption mechanism is even more complex, with the molecule approaching the surface first with one sulfonic group (position B), then two (position D), and finally arranging flat on the surface.

In both PS3 and PS4, the structures corresponding to the local minima of the PMF curves represent the molecules at an edge-on (more or less perpendicular) interaction with the surface. Even at this configuration, the edges of the molecules are at the optimum interaction distance with graphene $(\sim 0.34$ $\mathrm{nm}$ ). But as the molecules tilt to go to the fully adsorbed state they have to pass through energy barriers that appear at tilted configurations. Note that the presence of energy minima is underestimated by modelling just one molecule, because in the real system it is likely that the perpendicular configurations are further stabilized (namely we expect deeper local minima) by face-on van der Waals interaction with neighboring molecules.

Simulations show that the molecular asymmetry plays a central role in favouring the approach of the amphiphilic molecule toward graphene, displacing the water molecules
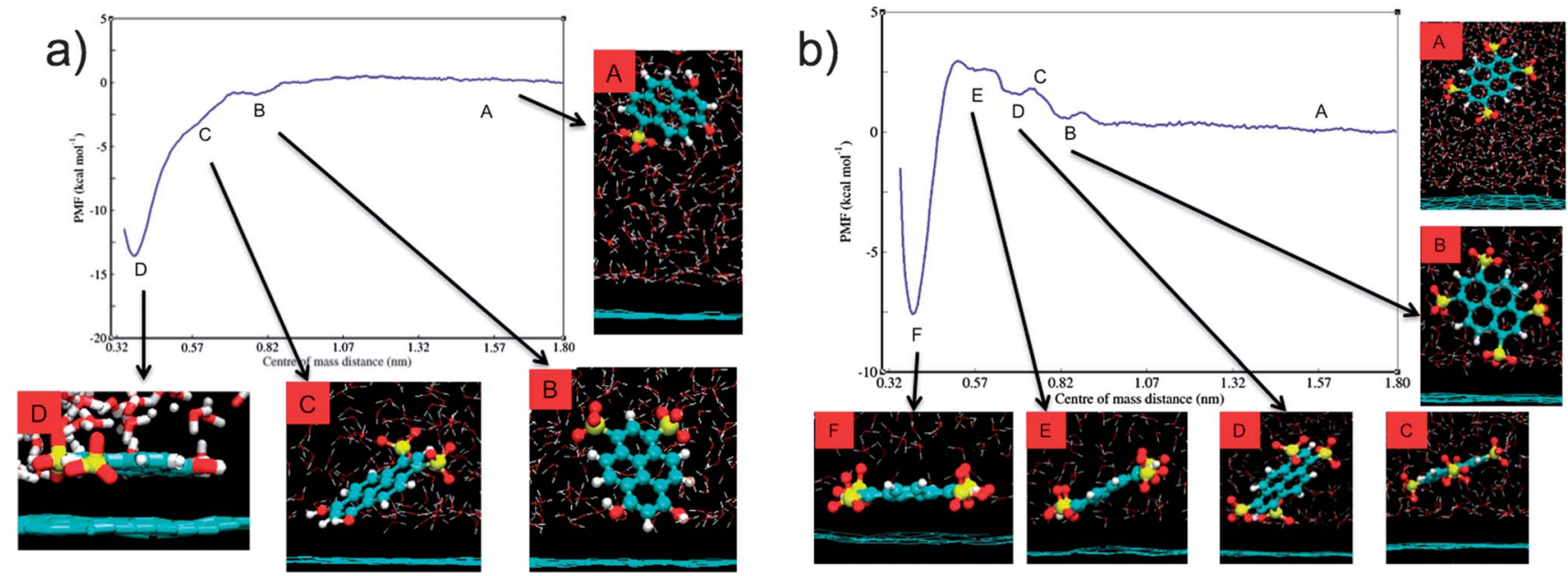

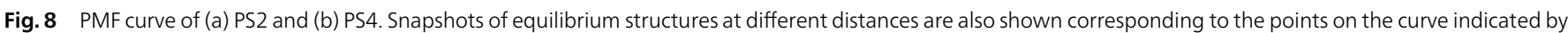
letters. 
present in between them (the presence of water molecules between the aromatic core of PS4 and the graphene surface is shown as an example in Fig. $8 \mathrm{~b}$ and $\mathrm{S} 4, \dagger$ with snapshots revealing the process as seen from below the graphene surface). Due to the asymmetric approach observed in Fig. 8, the water molecules initially present between the aromatic core and graphene at this stage are pushed laterally and removed in the subsequent steps, thus allowing maximal interaction. This "sliding" mechanism is similar, on a smaller scale, to what was recently postulated in simulations of the stacking of large graphene sheets in water, where the removal of an intercalated water layer is the ultimate step of the stacking process. ${ }^{46}$

The adsorption free energies of the four molecules determined from MD simulations correspond to the lowest minima in Fig. 7, and amount for each dye to $12.8 \mathrm{kcal} \mathrm{mol}^{-1}$ (PS1), 14.6 kcal $\mathrm{mol}^{-1}$ (PS2), $11.8 \mathrm{kcal} \mathrm{mol}^{-1}$ (PS3), and $8.4 \mathrm{kcal} \mathrm{mol}^{-1}$ (PS4). The adsorption free energy is determined by the interplay of the solvation free energy and the interaction free energy with the graphene surface. With increasing number of hydrophilic sulfonic groups, the solvation free energy of the molecules increases (see Table S3 in ESI $\dagger$ ), which in turn results in smaller (in magnitude) adsorption free energy. The sulfonic groups do not only interact with the solvent, but also screen the hydrophobic pyrene core in aqueous solution. Yet, instead of a regular decrease in adsorption free energy with increasing number of sulfonic groups, the MD simulations yield the largest adsorption energy for PS2, followed by PS1, PS3 and PS4.

To unravel the different factors influencing adsorption, the two-body interaction energies (energy difference between the full system and separated components, see Simulation details section) at the global minima on the PMF were analyzed further.

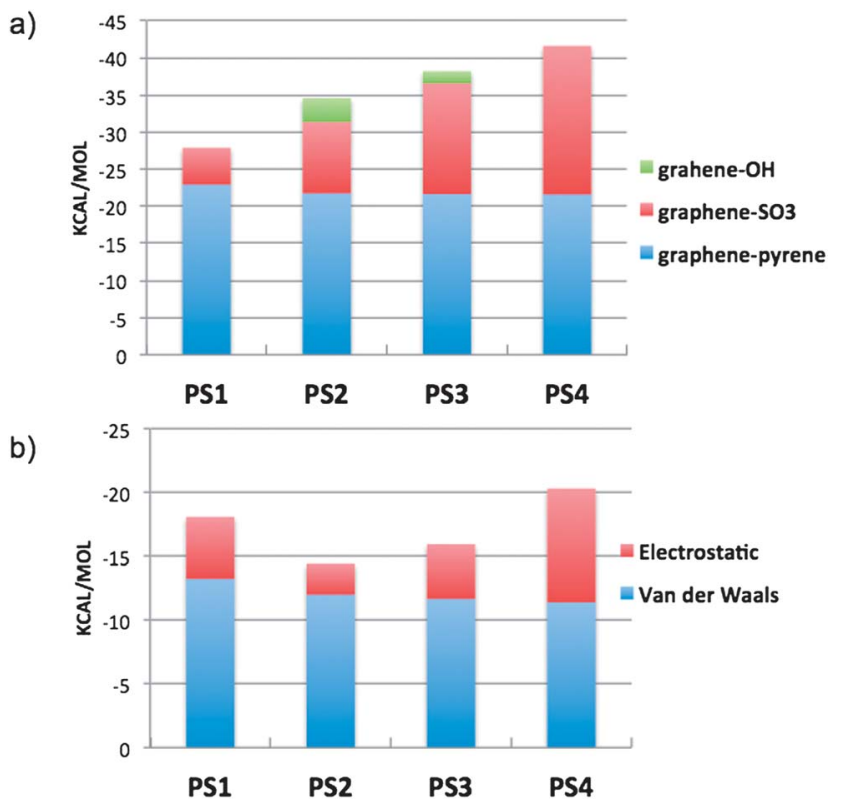

Fig. 9 (a) Contribution of the different components of the PSx dyes to the interaction energy of the molecules with graphene. (b) Electrostatic and van der Waals contributions of the pyrene core of adsorbed PSX molecules with the surrounding aqueous medium.
Fig. 9a displays the contributions to the interaction energy with the graphene substrate involving the aromatic core, the $-\mathrm{SO}_{3}$ and the $-\mathrm{OH}$ groups. The corresponding energy values together with the associated standard deviations are reported in Table S1 in ESI. $†$ We find that the interaction energy of the pyrene core with graphene as well as the contribution from each $-\mathrm{SO}_{3}$ unit to the overall interaction energy is about the same in all the dyes. (Table $\mathrm{S} 1$ in $\mathrm{ESI} \dagger$ reports also the total graphene- $\mathrm{SO}_{3}$ interaction normalized by the number of $-\mathrm{SO}_{3}$ groups for each dye.) Instead, it is the interaction of the pyrene core with the solvent (Fig. 9b) that varies in a less linear way, correlating well with the observed experimental exfoliation efficiency. As the - $\mathrm{OH}$ groups are grafted on the pyrene core (in going from PS1 to PS2), its interaction with water is less favored. Subsequently replacing the $-\mathrm{OH}$ by $-\mathrm{SO}_{3}$ moieties (in PS4) yields an increase in the interaction energy with water. The decomposition of this interaction energy into van der Waals and electrostatic interactions shows that the differences are mostly due to long-ranged electrostatic interactions that make PS4 too water-soluble to favour adsorption on graphene, while the pyrene moiety of PS2 shows the worst interaction with the solvent relative to the other dyes, making it more prone to stick to graphene sheets. The interaction of the functional groups $\left(-\mathrm{SO}_{3}\right.$ and $\left.-\mathrm{OH}\right)$ with the solvent is overwhelmingly high, as detailed in Tables $\mathrm{S} 2$ and $\mathrm{S} 3, \dagger$ suggesting that a major obstacle to the adsorption is due to solvent restructuring, in agreement with the PMF results.

\section{pH response: influence of molecular charges on graphene stability in suspensions}

As aforementioned, the dipole of PS2 and PS3 dyes changes significantly with $\mathrm{pH}$, influencing the optical and chemical properties of the dye. It is reasonable to assume that the large $\mathrm{pH}$ response will influence the exfoliation efficiency as well. We explored the effect of eventual charges present in correspondence of $-\mathrm{OH}$ groups by sonicating graphite with the respective dyes at different $\mathrm{pH}$. A systematic effect of $\mathrm{pH}$ could be observed on the total amount of suspended graphene. The $\mathrm{pH}$ effect on the absorption of dyes is portrayed in Fig. 10a. We used absorption spectroscopy to monitor (i) the amount of graphene material suspended at $\lambda=650 \mathrm{~nm}$ (Fig. 10b) and (ii) changes in pyrene protonation and charging at wavelengths between 200 and $500 \mathrm{~nm}$ (Fig. 11). For comparison, Fig. S1 in ESI $†$ shows the $\mathrm{pH}$ dependent UV spectra of pristine dye solutions.

A significant change in the amount of dissolved material was observed by varying the $\mathrm{pH}$ : the exfoliation efficiency is highest at neutral $\mathrm{pH}$ and decreases in strongly basic or acidic conditions (Fig. 9b). Acidic conditions induce a significantly more pronounced destabilization effect than basic conditions, for all four derivatives. Interestingly, this trend is also observed for pyrenes exposing only $-\mathrm{SO}_{3}$ moieties in the periphery such as PS1 and PS4, in which neither dye conformation nor light absorption is sensitive to $\mathrm{pH}$. Given that the addition of salt induces precipitation as well (as shown as an example by adding $\mathrm{Na}_{2} \mathrm{SO}_{4}$ to PS3, blue curve in Fig. 11), these results indicate an overall effect of ionic strength inducing destabilization, similarly to previous observations on reduced graphene oxide and 
a) GRAPHITE DISSOLUTION AT VARYING pH
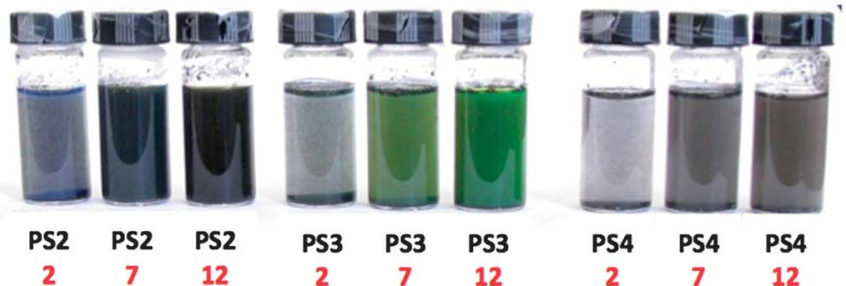

b)

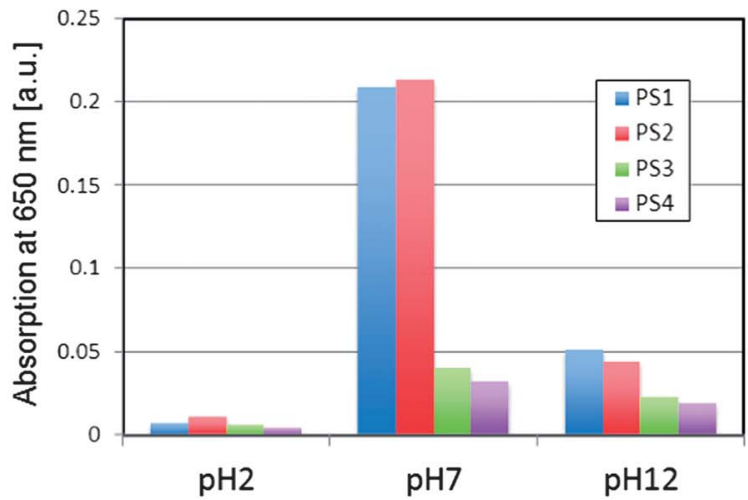

Fig. 10 (a) Image of the solutions obtained on sonicating graphite with the different dyes at $\mathrm{pH}=2,7$ and 10 . $\mathrm{pH}$ was adjusted to $\mathrm{pH}=2$ and $\mathrm{pH}=12 \mathrm{by}$ adding $\mathrm{HCl}$ and $\mathrm{NaOH}$. Initial dye concentration $3 \times 10^{-4} \mathrm{~mol} \mathrm{~L}^{-1}$, sonicated for 4 hours. (b) Absorbance at $650 \mathrm{~nm}$ for all the solutions at different pH shown in (a) (solutions diluted $1: 20$ for measurement).

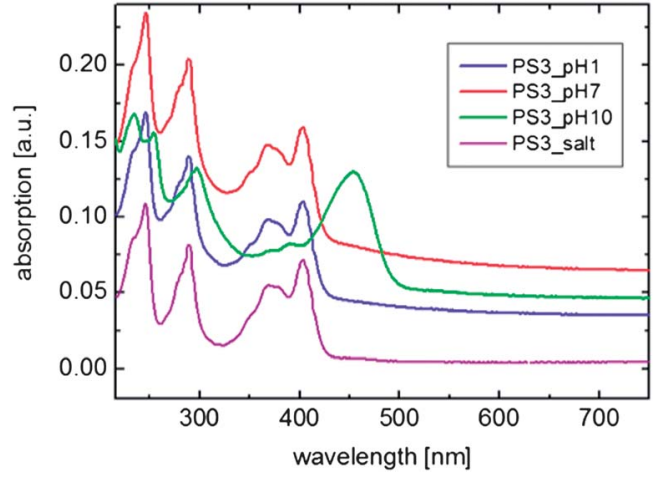

Fig. 11 Typical changes in absorption, in this case for PS3 dye at $c=7 \times 10^{-5}$ mol $\mathrm{L}^{-1}$, for exfoliation at different $\mathrm{pH}$ values and for addition of salt (solutions diluted $1: 20$ for measurement).

graphene sheets stabilized by surfactants containing carbonyl groups. ${ }^{47}$ As expected for the pH indicators PS2 and PS3, the absorption band changes, because acidic conditions give rise to a blue shifted absorption band for the deprotonated species. ${ }^{48}$ Although the change in the protonation state can be clearly observed in the presence of graphene, the protonation state is found to be not relevant for the ability of molecules to stabilize graphene sheets in suspension: the same trend in $\mathrm{pH}$ dependent suspension efficiency is observed for all the common pyrene sulfonic acid derivatives with and without hydroxyl functionalization, with the highest efficiency in neutral, decreased graphene concentration in basic and heavy precipitation in acidic conditions (Fig. 10).

\section{Discussion}

The screening effect of molecules on graphene-graphene stacking interactions has been previously studied at the molecular scale, showing that the main factor hindering aggregation is the presence of a layer of molecules adsorbed on the graphene sheets; atomic scale modelling has been used to reproduce the stabilizing effect of solvents and surfactants on graphene sheets. ${ }^{36,49}$ Empirical, mean field approaches have been developed to correlate exfoliation efficiency to solubility parameters quantifying the dispersive, polar, and hydrogen bonding properties of the solvent, or more simply to the surface tension or the refractive index of solvents (for an extensive review, see ref. 50). In case of graphite exfoliation into solutions of aliphatic or aromatic surfactants, complex processes may take place, which involve the formation of charged graphenemolecule complexes, and even charge or energy transfer between the molecules and graphene..$^{51,52}$

The results obtained here show that the true picture is more complex than those reported in previous works, ${ }^{31,32,37}$ with a relevant interaction of both the aromatic and polar moieties with the graphene surface. UV/VIS absorption data reveal that PS2 yielded the highest amount of exfoliated material in suspension, before and after purification, with PS2 being the derivative with the largest adsorption energy on graphene and the highest molecular dipole in water, as demonstrated by MD simulations.

For effective interaction, the aromatic cores of pyrene and graphene have to overcome the steric repulsions originating from a confined single layer of solvent, similarly to what was 
observed for the re-aggregation process of large, parallel graphene sheets. ${ }^{49}$ The molecular dipole of PS2 is thus not important per se; instead, an asymmetry in polarity can facilitate the "sliding" of the molecule into the solvent layer, and the lateral displacement of the water molecules confined between the aromatic core and the graphene surface, removing the energy barrier to be overcome for dye adsorption.

A similar mechanism takes place for the monosubstitued derivative PS1, where, however, the lower polarity of the molecule decreases the graphene solubilization effect. With PS3 and PS4, the presence of polar groups on both sides of the pyrene moiety hinders the release of the confined water, thus hindering the molecular approach to graphene. As highly functionalized PS3 and PS4 derivatives are expected to exhibit stronger solvent interaction, the solvent layer may also be strongly localized, rendering non-covalent functionalization of graphene less favourable. The combination of molecular polarity, molecular dipole and adsorption mechanism thus reveals PS2 being the most effective molecule for graphene solubilization among the four dyes studied.

Noteworthy, a different ranking was observed for "dye capture" and exfoliation experiments, suggesting that the adsorption on bulk graphite is governed to a less extent by the interaction between the dye and graphene than the exfoliation process. The adsorption on graphite surfaces is simply inversely proportional to the number of sulfonic groups present on the pyrene core and thus to molecule solvation energy. Instead, exfoliation relies more on an efficient interaction of the dye with the graphene surface.

The exfoliation efficiency changes significantly with $\mathrm{pH}$, yielding lower graphene concentrations in acidic and basic conditions. The $\mathrm{pH}$ dependent exfoliation efficiency is not related to the protonation or deprotonation of the $\mathrm{pH}$ sensitive molecules, as could be expected. All suspensions, even the ones based on molecules without $\mathrm{pH}$ sensitive hydroxyl groups, show a loss in stability in both high and low $\mathrm{pH}$ conditions. Destabilization was also observed in the presence of salts. A similar $\mathrm{pH}$ dependence is well documented with common aliphatic surfactants such as SDBS. ${ }^{4}$ The suspended graphene-pyrene composite studied here, once exfoliated, can be considered in a good approximation as suspensions stabilized by electrostatic repulsion, comparable to micelle stabilized particles, even if the mechanism of colloidal stabilization is not based on micelle formation, but on the interaction of the aromatic molecule with the graphene lattice. Even if the functionalization of the pyrene core plays a major role in the graphene yield obtained with the respective molecule, an additional effect of protonation state with the $\mathrm{pH}$ sensitive derivatives PS2 and PS3 was not observed.

\section{Conclusions}

We compared four pyrene derivatives with varying number of polar functionalizations for their efficiency as exfoliation agents in the preparation of stable, aqueous graphene suspensions with liquid phase exfoliation. We found that the four studied derivatives exfoliate graphite, yielding stable suspensions in water. A relevant fraction of monolayer graphene was obtained in all samples, but AFM and TEM show that a layer of molecules covers the sheets. The total concentration of suspended graphene depends significantly on the polar functionalization present on the pyrene core.

The concentration of graphene exfoliated and dissolved from bulk graphite to suspension is highest for the PS2 derivative, the latter having the largest dipole and most asymmetric functionalization. The adsorption of the molecules from solution to bulk graphite is simply inversely proportional to the number of highly polar sulfonic groups present. Molecular dynamic calculation revealed that a critical factor in the interaction of pyrene derivatives with graphene involves a thin solvent layer confined between the dye and the graphene surface; the amphiphilic molecule changes its orientation when approaching the surface to slide into this layer, and the asymmetric shape of PS2 molecule facilitates this step. Simulations indicate that the molecular dipole is thus not important per se, but because it facilitates the "sliding" of the molecule into the solvent layer, and therefore the lateral displacement of the water molecules collocated between the aromatic cores of the dye and the graphene substrate.

Once the molecules are adsorbed on graphene, these graphene-organic hybrids yield stable suspensions in water. The stability of the suspensions is highly $\mathrm{pH}$ responsive for all four derivatives, and the solubility trend observed with varying $\mathrm{pH}$ is the same for the different functionalizations of the pyrene core: in these graphene organic hybrid systems, colloidal stabilization is achieved through electrostatic repulsion between charges introduced by the surfactant and can be overcome by changing $\mathrm{pH}$ or adding salts. The prepared graphene can be considered in a good approximation as suspensions stabilized by electrostatic repulsion.

\section{Experimental details}

Pyrene derivatives and graphite powder were obtained from Aldrich, and dissolved in ultrapure water using high power sonication in a water bath (Hielscher UP 50H). Pyrene concentration was $3.3 \times 10^{-4} \mathrm{~mol} \mathrm{~L}^{-1}$ in all cases. Different previous works show the advantages of long sonication time (until $400 \mathrm{~h}$ ) for exfoliation of graphene in water ${ }^{53}$ or in other solvents. ${ }^{54}$ The highest concentration of dispersed graphene can be achieved by increasing the sonication time, and this can facilitate the comparison of different derivatives especially for molecules with different kinetics of exfoliation.

The suspensions of four derivatives were processed in the same way at the same time to reduce the variables related to the method like temperature, time, power etc. Absorption spectra were recorded using a Perkin-Elmer Lambda 20 spectrometer. AFM measurements were carried out using a Digital Instruments AFM (NT-MDT), using Nanoprobe cantilevers (model: RTESP, material: 1-10 Ohm cm phosphorus (n) doped Si, f0: $27-309 \mathrm{kHz}$, k: $20-80 \mathrm{~N} \mathrm{~m}^{-1}$; from Veeco) operating in tapping mode.

The Scanning Electron Microscopy (SEM) images were obtained with a ZEISS 1530 instrument. Transmission Electron Microscopy (TEM) observations were carried out with a Fei Tecnai F20 TEM equipped with a Schottky emitter and operating at $80 \mathrm{keV}$. 
Raman measurements were performed using a Witec alpha300 Raman spectrometer, equipped with 488, 514.5 and 633 $\mathrm{nm}$ excitation lines and a piezoelectric stage for Raman mapping.

\section{Simulation details}

The process of adsorption of the pyrene derivative molecules on the surface of graphene in a water medium was simulated using Molecular Dynamics simulations.

The OPLS-AA force field ${ }^{55}$ was parameterized for the sulfonic groups of the molecules by fitting charges and dihedral angle parameters from electronic structure calculations with the b3lyp/ $6-31 \mathrm{~g}(\mathrm{~d}, \mathrm{p})^{56,57}$ level of theory. For the rest of the systems, we used the existing parameters of the OPLS-AA force field, as implemented in the GROMACS 4.0.7 software package. ${ }^{58}$ A simulation box of size $2.8 \times 2.8 \times 7 \mathrm{~nm}^{-3}$, containing an infinite sheet of graphene at the bottom, was used for each pyrene derivative. Each molecule was restrained at a perpendicular distance of $1.8 \mathrm{~nm}$ from the plane of the graphene sheet and the box was filled with water molecules. The standard $\mathrm{SPC} / \mathrm{E}^{59}$ model was used to simulate the water molecules. The STTLE ${ }^{60}$ algorithm was used to constrain the bond lengths in the water molecules, while the other bonds were constrained using the $\operatorname{LINCS}^{61,62}$ algorithm. A steepest decent energy minimization with 2 fs step size was done on each simulation system followed by a 50 ps MD run, under the NVT ensemble, to ensure further relaxation.

For each system, an umbrella pulling simulation technique ${ }^{58}$ was used to calculate the potential of mean force (PMF) of the adsorption process. 51 to 73 sampling windows were used depending on the simulation system as required to ensure stable results. In each window (at various separation distances between the center of mass of the molecule and the graphene surface), the molecule was restrained with a harmonic force and a 100 ps equilibration MD simulation was performed, under the NPT ensemble, followed by a 50 ns production run. While restrained within a window, the molecule was allowed to rotate around its center of mass and translate along the $x y$ plane (parallel to the plane of the graphene sheet). The interaction forces required to keep the molecule at distances from the surface were integrated to calculate the PMF at various distances, using the weighted histogram analysis method (WHAM) ${ }^{63,64}$ Only the last 25 ns of the production simulation were used for this analysis. The free energy of adsorption of the molecule, which is the difference between the PMF at the farthest distance $(1.8 \mathrm{~nm}$ ) and that of the fully adsorbed (global minimum energy) state, was determined from the resulting PMF curve. In addition, the two-body interaction energies involving the different parts of the dyes with the graphene and the solvent were calculated. These were computed at the global minimum geometry along the PMF as the energy difference between the dye plus graphene [dye plus solvent] and the sum of the individual graphene [solvent] and dye.

Each MD simulation mentioned above (NVT or NPT), was simulated at a temperature of $298 \mathrm{~K}$ and a pressure of $1 \mathrm{bar}$ with a time step of $2 \mathrm{fs}$. The PME algorithm was used for electrostatic interactions, with a cut-off distance of $1.2 \mathrm{~nm}$. A single cut-off distance of $1.2 \mathrm{~nm}$ was also used for van der Waals (vdW) interactions. The Nose-Hoover ${ }^{65}$ algorithm was used as the thermostat, while pressure was equilibrated using the Parrinello-Rahman ${ }^{66}$ algorithm. Periodic boundary conditions in the three directions were maintained in all cases.

\section{Acknowledgements}

This work was supported by the European Science Foundation (ESF) under the EUROCORES Program EuroGRAPHENE (GOSPEL), the Operative Program FESR 2007-2013 of Regione Emilia-Romagna - Attività I.1.1. and the EC Marie-Curie ITNGENIUS (PITN-GA-2010-264694). Supports from the International Center for Frontier Research in Chemistry (icFRC) are also gratefully acknowledged. The work in Mons was also supported by the European project MMM@HPC (FP7-RI-261594), the Interuniversity Attraction Pole program of the Belgian Federal Science Policy Office (PAI 6/27), Programme d'Excellence de la Région Wallonne (OPTI2MAT project), and FNRSFRFC. D.B. is a FNRS Research Fellow.

\section{References}

1 Y. Hernandez, V. Nicolosi, M. Lotya, F. M. Blighe, Z. Y. Sun, S. De, I. T. McGovern, B. Holland, M. Byrne, Y. K. Gun'ko, J. J. Boland, P. Niraj, G. Duesberg, S. Krishnamurthy, R. Goodhue, J. Hutchison, V. Scardaci, A. C. Ferrari and J. N. Coleman, Nat. Nanotechnol., 2008, 3, 563-568.

2 M. Lotya, Y. Hernandez, P. J. King, R. J. Smith, V. Nicolosi, L. S. Karlsson, F. M. Blighe, S. De, Z. M. Wang, I. T. McGovern, G. S. Duesberg and J. N. Coleman, J. Am. Chem. Soc., 2009, 131, 3611-3620.

3 L. Guardia, M. J. Fernandez-Merino, J. I. Paredes, P. SolisFernandez, S. Villar-Rodil, A. Martinez-Alonso and J. M. D. Tascon, Carbon, 2011, 49, 1653-1662.

4 R. J. Smith, M. Lotya and J. N. Coleman, New J. Phys., 2010, 12, 125008.

5 J. W. T. Seo, A. A. Green, A. L. Antaris and M. C. Hersam, J. Phys. Chem. Lett., 2011, 2, 1004-1008.

6 N. Behabtu, J. R. Lomeda, M. J. Green, A. L. Higginbotham, A. Sinitskii, D. V. Kosynkin, D. Tsentalovich, A. N. G. ParraVasquez, J. Schmidt, E. Kesselman, Y. Cohen, Y. Talmon, J. M. Tour and M. Pasquali, Nat. Nanotechnol., 2010, 5, 406-411.

7 C. Valles, C. Drummond, H. Saadaoui, C. A. Furtado, M. He, O. Roubeau, L. Ortolani, M. Monthioux and A. Penicaud, J. Am. Chem. Soc., 2008, 130, 15802.

8 Q. H. Wang and M. C. Hersam, Nat. Chem., 2009, 1, 206-211. 9 H. Sirringhaus, P. J. Brown, R. H. Friend, M. M. Nielsen, K. Bechgaard, B. M. W. Langeveld-Voss, A. J. H. Spiering, R. A. J. Janssen, E. W. Meijer, P. Herwig and D. M. de Leeuw, Nature, 1999, 401, 685-688.

10 R. Capelli, S. Toffanin, G. Generali, H. Usta, A. Facchetti and M. Muccini, Nat. Mater., 2010, 9, 496-503.

11 L. Schmidt-Mende, A. Fechtenkotter, K. Mullen, E. Moons, R. H. Friend and J. D. MacKenzie, Science, 2001, 293, 1119-1122. 12 J. Nelson, Curr. Opin. Solid State Mater. Sci., 2002, 6, 87-95. 13 J. Y. Kim, K. Lee, N. E. Coates, D. Moses, T. Q. Nguyen, M. Dante and A. J. Heeger, Science, 2007, 317, 222-225. 
14 N. S. Sariciftci, L. Smilowitz, A. J. Heeger and F. Wudl, Science, 1992, 258, 1474-1476.

$15 \mathrm{~J}$. Li, M. Kastler, W. Pisula, J. W. F. Robertson, D. Wasserfallen, A. C. Grimsdale, J. Wu and K. Müllen, Adv. Funct. Mater., 2007, 17, 2528-2533.

16 A. Facchetti, Mater. Today, 2007, 10, 28-37.

17 A. C. Arias, J. D. MacKenzie, I. McCulloch, J. Rivnay and A. Salleo, Chem. Rev., 2010, 110, 3-24.

18 R. Hamilton, J. Smith, S. Ogier, M. Heeney, J. E. Anthony, I. McCulloch, J. Veres, D. D. C. Bradley and T. D. Anthopoulos, Adv. Mater., 2009, 21, 1166-1171.

19 R. Ionescu, Y. Broza, H. Shaltieli, D. Sadeh, Y. Zilberman, X. Feng, L. Glass-Marmor, I. Lejbkowicz, K. Mullen, A. Miller and H. Haick, ACS Chem. Neurosci., 2011, 2, 687-693. 20 K. Müllen and J. P. Rabe, Acc. Chem. Res., 2008, 41, 511-520. 21 A. C. Grimsdale and K. Müllen, Angew. Chem., Int. Ed., 2005, 44, 5592-5629.

22 H. N. Tsao, W. Pisula, Z. H. Liu, W. Osikowicz, W. R. Salaneck and K. Mullen, Adv. Mater., 2008, 20, 2715-2719.

23 B. A. Jones, M. J. Ahrens, M. H. Yoon, A. Facchetti, T. J. Marks and M. R. Wasielewski, Angew. Chem., Int. Ed., 2004, 43, 6363-6366.

24 T. M. Pappenfus, R. J. Chesterfield, C. D. Frisbie, K. R. Mann, J. Casado, J. D. Raff and L. L. Miller, J. Am. Chem. Soc., 2002, 124, 4184-4185.

25 A. L. Briseno, S. C. B. Mannsfeld, C. Reese, J. M. Hancock, Y. Xiong, S. A. Jenekhe, Z. Bao and Y. Xia, Nano Lett., 2007, 7, 2847-2853.

26 H. Z. Chen, M. M. Ling, X. Mo, M. M. Shi, M. Wang and Z. Bao, Chem. Mater., 2007, 19, 816-824.

27 J. H. Oh, S. Liu, Z. Bao, R. Schmidt and F. Würthner, Appl. Phys. Lett., 2007, 91, 212107.

28 R. Schmidt, M. M. Ling, J. H. Oh, M. Winkler, M. Konemann, Z. N. Bao and F. Würthner, Adv. Mater., 2007, 19, 3692-3695.

29 J. H. Jang, D. Rangappa, Y. U. Kwon and I. Honma, J. Mater. Chem., 2011, 21, 3462-3466.

30 X. C. Dong, C. Y. Su, W. J. Zhang, J. W. Zhao, Q. D. Ling, W. Huang, P. Chen and L. J. Li, Phys. Chem. Chem. Phys., 2010, 12, 2164-2169.

31 M. Zhang, R. R. Parajuli, D. Mastrogiovanni, B. Dai, P. Lo, W. Cheung, R. Brukh, P. L. Chiu, T. Zhou, Z. F. Liu, E. Garfunkel and H. X. He, Small, 2010, 6, 1100-1107.

32 X. H. An, T. J. Simmons, R. Shah, C. Wolfe, K. M. Lewis, M. Washington, S. K. Nayak, S. Talapatra and S. Kar, Nano Lett., 2010, 10, 4295-4301.

33 Y. X. Xu, H. Bai, G. W. Lu, C. Li and G. Q. Shi, J. Am. Chem. Soc., 2008, 130, 5856.

34 M. Cardinali, L. Valentini and J. M. Kenny, J. Phys. Chem. C, 2011, 115, 16652-16656.

35 S. Bose, T. Kuila, A. K. Mishra, N. H. Kim and J. H. Lee, Nanotechnology, 2011, 22, 405603.

36 S. C. Lin, C. J. Shih, M. S. Strano and D. Blankschtein, J. Am. Chem. Soc., 2011, 133, 12810-12823.

37 D. Parviz, S. Das, H. S. T. Ahmed, F. Irin, S. Bhattacharia and M. J. Green, ACS Nano, 2012, 6, 8857-8867.

38 V. Palermo and P. Samori, Angew. Chem., Int. Ed., 2007, 46, 4428-4432.
39 H. Yang, Y. Hernandez, A. Schlierf, A. Felten, A. Eckmann, S. Johal, P. Louette, J. J. Pireaux, X. Feng, K. Mullen, V. Palermo and C. Casiraghi, Carbon, 2012, 53, 357-365, DOI: 10.1016/j.carbon.2012.1011.1022.

40 A. Hakonen and S. Hulth, Talanta, 2010, 80, 1964-1969.

41 D. C. Dong and M. A. Winnik, Can. J. Chem., 1984, 62, 25602565.

42 M. Ottonelli, M. Piccardo, D. Duce, S. Thea and G. Dellepiane, J. Phys. Chem. A, 2012, 116, 611-630.

43 X. Y. Pan, H. Li, K. T. Nguyen, G. Gruner and Y. L. Zhao, J. Phys. Chem. C, 2012, 116, 4175-4181.

44 S. Detriche, S. Devillers, J. F. Seffer, J. B. Nagy, Z. Mekhalif and J. Delhalle, Carbon, 2011, 49, 2935-2943.

45 R. Colle, G. Grosso, A. Ronzani, M. Gazzano and V. Palermo, Carbon, 2011, 50, 1332-1337.

$46 \mathrm{~W}$. Lv and R. Wu, http://arxiv.org/ftp/arxiv/papers/1211/ 1211.2094.pdf.

47 F. Yang, Y. Q. Liu, L. A. Gao and J. Sun, J. Phys. Chem. C, 2010, 114, 22085-22091.

48 G. Velasquez, M. S. Ureta-Zanartu, C. Lopez-Alarcon and A. Aspee, J. Phys. Chem. B, 2011, 115, 6661-6667.

49 C. J. Shih, S. C. Lin, M. S. Strano and D. Blankschtein, J. Am. Chem. Soc., 2010, 132, 14638-14648.

50 J. N. Coleman, Adv. Funct. Mater., 2009, 19, 3680-3695.

51 N. V. Kozhemyakina, J. M. Englert, G. A. Yang, E. Spiecker, C. D. Schmidt, F. Hauke and A. Hirsch, Adv. Mater., 2010, 22, 5483-5487.

52 R. S. Swathi and K. L. Sebastian, J. Chem. Phys., 2008, 129, 054703.

53 M. Lotya, P. J. King, U. Khan, S. De and J. N. Coleman, ACS Nano, 2010, 4, 3155-3162.

54 A. O'Neill, U. Khan, P. N. Nirmalraj, J. Boland and J. N. Coleman, J. Phys. Chem. C, 2011, 115, 5422-5428.

55 W. L. Jorgensen, D. S. Maxwell and J. TiradoRives, J. Am. Chem. Soc., 1996, 118, 11225-11236.

56 A. D. Becke, J. Chem. Phys., 1993, 98, 5648.

57 C. Lee, W. Yang and R. G. Parr, Phys. Rev. B: Condens. Matter Mater. Phys., 1988, 37, 785.

58 GROMACS, D. van der Spoel, E. Lindahl, B. Hess, A. R. van Buuren, E. Apol, P. J. Meulenhoff, D. P. Tieleman, A. L. T. M. Sijbers, K. A. Feenstra, R. van Drunen and H. J. C. Berendsen, Gromacs User Manual Version 4.0, http://www.gromacs.org, 2005.

59 H. J. C. Berendsen, J. R. Grigera and T. P. Straatsma, J. Phys. Chem., 1987, 91, 6269-6271.

60 S. Miyamoto and P. A. Kollman, J. Comput. Chem., 1992, 13, 952-962.

61 B. Hess, H. Bekker, H. J. C. Berendsen and J. G. E. M. Fraaije, J. Comput. Chem., 1997, 18, 1463-1472.

62 B. Hess, J. Chem. Theory Comput., 2008, 4, 116-122.

63 A. M. Ferrenberg and R. H. Swendsen, Phys. Rev. Lett., 1989, 63, 1195.

64 S. Kumar, D. Bouzida, R. H. Swendsen, P. A. Kollman and J. M. Rosenberg, J. Comput. Chem., 1992, 13, 101.

65 W. G. Hoover, Phys. Rev. A, 1985, 31, 1695.

66 M. Parrinello and A. Rahman, J. Appl. Phys., 1981, 52, 7182. 